\title{
Fatty acids from fat cell lipolysis do not activate an inflammatory response but are stored as triacylglycerols in adipose tissue macrophages
}

\author{
Sylvie Caspar-Bauguil ${ }^{1,2,3}$ - Catherine-Ines Kolditz ${ }^{1,2}$ - Corinne Lefort $^{1,2}$ •

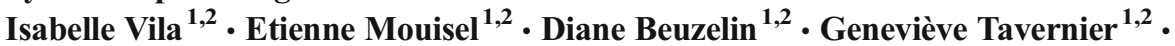 \\ Marie-Adeline Marques ${ }^{1,2}$ - Alexia Zakaroff-Girard ${ }^{2,4,5}$ - Christiane Pecher $^{2,4}$. \\ Marianne Houssier ${ }^{1,2} \cdot$ Lucile Mir $^{1,2}$ - Sarah Nicolas ${ }^{1,2} \cdot$ Cédric Moro $^{1,2}$ • \\ Dominique Langin ${ }^{1,2,3}$
}

Received: 25 June 2015 / Accepted: 9 July 2015 /Published online: 6 August 2015

(C) Springer-Verlag Berlin Heidelberg 2015

\begin{abstract}
Aims/hypothesis Activation of macrophages by fatty acids (FAs) is a potential mechanism linking obesity to adipose tissue (AT) inflammation and insulin resistance. Here, we investigated the effects of FAs released during adipocyte lipolysis on AT macrophages (ATMs).

Methods Human THP-1 macrophages were treated with media from human multipotent adipose-derived stem (hMADS) adipocytes stimulated with lipolytic drugs. Macrophages were also treated with mixtures of FAs and an inhibitor of Toll-like receptor 4 , since this receptor is activated by saturated FAs.
\end{abstract}

Sylvie Caspar-Bauguil, Catherine-Ines Kolditz and Corinne Lefort contributed equally to this study.

Electronic supplementary material The online version of this article (doi:10.1007/s00125-015-3719-0) contains peer-reviewed but unedited supplementary material, which is available to authorised users.

Dominique Langin

dominique.langin@inserm.fr

1 Inserm, UMR1048, Institute of Metabolic and Cardiovascular Diseases, I2MC, Obesity Research Laboratory, Team 4, CHU Rangueil, 1 avenue Jean Poulhès, BP 84225, 31432 Toulouse Cedex 4, France

2 University of Toulouse, UMR1048, Paul Sabatier University, Toulouse, France

3 Toulouse University Hospitals, Department of Clinical Biochemistry, Toulouse, France

4 Inserm, UMR1048, Cytometry Facility, Institute of Metabolic and Cardiovascular Diseases, Toulouse, France

5 Inserm, UMR1048, Team 1, Institute of Metabolic and Cardiovascular Diseases, Toulouse, France
Levels of mRNA and the secretion of inflammation-related molecules were measured in macrophages. FA composition was determined in adipocytes, conditioned media and macrophages. The effect of chronic inhibition or acute activation of fat cell lipolysis on ATM response was investigated in vivo in mice.

Results Whereas palmitic acid alone activates THP-1, conditioned media from hMADS adipocyte lipolysis had no effect on IL, chemokine and cytokine gene expression, and secretion by macrophages. Mixtures of FAs representing de novo lipogenesis or habitual dietary conditions also had no effect. FAs derived from adipocyte lipolysis were taken up by macrophages and stored as triacylglycerol droplets. In vivo, chronic treatment with an antilipolytic drug did not modify gene expression and number of ATMs in mice with intact or defective Tlr4. Stimulation of adipocyte lipolysis increased storage of neutral lipids by macrophages without change in number and phenotype.

Conclusions/interpretation Our data suggest that adipocyte lipolysis does not activate inflammatory pathways in ATMs, which instead may act as scavengers of FAs.

Keywords Adipocyte - Adipose tissue - Denovolipogenesis · Fatty acids · Inflammatory response · Insulin resistance · Lipolysis · Macrophage · Triacylglycerol · TLR4

$\begin{array}{ll}\text { Abbreviations } \\ \text { AT } & \text { Adipose tissue } \\ \text { ATMs } & \text { Adipose tissue macrophages } \\ \text { FAs } & \text { Fatty acids } \\ \text { hMADS } & \text { human multipotent adipose-derived stem }\end{array}$




$\begin{array}{ll}\text { HSLi } & \text { Hormone-sensitive lipase inhibitor } \\ \text { LPS } & \text { Lipopolysaccharide } \\ \text { MCP1 } & \text { Monocyte chemoattractant protein 1 } \\ \text { NF-kB } & \text { Nuclear factor-kB } \\ \text { TAG } & \text { Triacylglycerol } \\ \text { TLR4 } & \text { Toll-like receptor } 4 \\ \text { WT } & \text { Wild-type }\end{array}$

\section{Introduction}

Chronic low-grade inflammation is associated with insulin resistance and obesity-related metabolic disorders such as type 2 diabetes mellitus [1,2]. This inflammatory state appears to initiate in adipose tissue (AT), as immune cell infiltration in AT has been shown in rodent models to take place gradually during the time course of obesity prior to the development of insulin resistance [3, 4]. Notably, infiltrated AT macrophages (ATMs) represent a major source of proinflammatory cytokines and chemokines, which may impair insulin sensitivity $[1,2,5,6]$ and interfere with adipocyte functions [7, 8], thereby aggravating the metabolic dysfunction of AT. However, excessive release of fatty acids (FAs) from expanded AT has been hypothesised to cause insulin resistance $[9,10]$. Thus, there is growing evidence that both adipocytes and ATMs contribute to the development of insulin resistance. Crosstalk between the two cell types may play a key role in this process.

The metabolic factors regulating the ATM immune response remain poorly defined. Saturated FAs exert proinflammatory effects via the endotoxin receptor, Toll-like receptor 4 (TLR4), expressed in ATMs [11-15]. We, therefore, hypothesised that FAs released by stimulation of human adipocyte lipolysis could promote activation of inflammatory pathways in ATMs through TLR4 signalling. To test this hypothesis, conditioned media were prepared from human adipocytes stimulated with lipolytic and antilipolytic drugs and used to treat human macrophages. Human macrophages were also treated with mixtures of FAs similar to that in the lipolytic medium or representative of human AT in normal dietary conditions. The effect of acute activation or chronic inhibition of lipolysis on AT inflammation was investigated in vivo in mice expressing functional or defective TLR4 signalling.

\section{Methods}

Cell culture and lipolysis Human multipotent adiposederived stem (hMADS) cells provided by G. Ailhaud and E-Z Amri (CNRS, University of Nice-Sophia Antipolis, Nice, France) were cultured and differentiated as previously described [16]. Lipolytic challenges were performed by treating adipocytes for $3 \mathrm{~h}$ with forskolin (Sigma-Aldrich, Saint-Louis,
MO, USA), isoprenaline (Sigma-Aldrich), OPC3911 (Otsuka Pharmaceutical, Tokyo, Japan) [17] and/or BAY 59-9435 (NoValix, Illkirch, France) [18]. The same treatments were carried out in parallel in cell-free wells used as controls of hMADS conditioned media for THP-1 cell treatment. Glycerol and NEFA released in the medium were measured using free glycerol reagent (Sigma-Aldrich) and NEFA-HR (2) from Wako Chemicals (Neuss, Germany). Protein concentrations were determined with Bio-Rad (Marnes-la-Coquette, France) protein assay using BSA as standard. THP-1 cells (TIB202, American Type Culture Collection, Teddington, UK) were routinely cultured in RPMI 1640 medium (Gibco, Grand Island, NY, USA) supplemented with $10 \%$ vol./vol. FBS. Cell lines are checked for mycoplasma contamination using Hoechst 33342 (Invitrogen, Paisley, UK). See the electronic supplementary material (ESM) Methods for further details.

Fatty acid mixtures Mixtures of FAs were prepared to mimic the composition of hMADS lipolysis conditioned media or a profile typical of human adipocyte lipolysis [19] (ESM Table 1).

THP-1 cell treatments THP- 1 cells were treated for $72 \mathrm{~h}$ in the presence of $2 \%$ BSA with hMADS conditioned media or exogenous FAs. Culture supernatant fractions and cell extracts were collected and stored at $-80^{\circ} \mathrm{C}$ until use. TAK-242, a specific inhibitor of the TLR4 signalling pathway, was kindly provided by GlaxoSmithKline (Les Ulis, France).

Gas chromatography analysis of triacylglycerol FAs After washing, cells were suspended in PBS. Lipid extraction was performed using the Folch method and FA composition was determined by capillary gas chromatography [20].

Bodipy staining of neutral lipids Cells were fixed with paraformaldehyde solution and non-specific interactions were blocked by $1 \%$ BSA. Lipid droplets and nuclei were stained with a Bodipy neutral lipid probe 493/503 (Invitrogen) and Hoechst 33342 (Invitrogen) in PBS for $30 \mathrm{~min}$. Cells were then washed twice with PBS and visualised under reverse fluorescent microscopy.

Cytokine quantification IL-6, IL-10 and monocyte chemoattractant protein 1 (MCP1) were measured in cell culture supernatant fractions using ELISA kits (IL-6, Invitrogen; MCP1, Ray Biotech, Norcross, GA, USA; IL-10, Enzo Life Sciences, Farmingdale, NY, USA).

Animal studies Animal protocols were performed in accordance with French and European Animal Care Facility guidelines. Mice were housed at constant temperature $\left(20-22^{\circ} \mathrm{C}\right)$ and humidity (50-60\%) conditions, with a $12 / 12 \mathrm{~h}$ light/dark cycle (lights on at 07:00 hours) and given free access to food. Five-week-old male $\mathrm{C} 3 \mathrm{H} / \mathrm{HeJ}$ (stock 000659) mice (which 
show defective TLR4 signalling) and wild-type (WT) $\mathrm{C} 3 \mathrm{H} /$ $\mathrm{HeOuJ}$ (stock 000635) male mice were purchased from the Jackson Laboratory (Bar Harbor, ME, USA). After weaning, $\mathrm{C} 3 \mathrm{H} / \mathrm{HeJ}$ and $\mathrm{C} 3 \mathrm{H} / \mathrm{HeOuJ}$ mice were fed a high-fat $\operatorname{diet}(45 \%$ energy as fat, Research Diets D12451, New Brunswick, NJ, USA) for 8 weeks. Treatment with the antilipolytic drug BAY $59-9435$ at $70 \mathrm{mg} / \mathrm{kg}$ or vehicle was given orally once a day in the last 2 weeks of diet. For in vivo lipolytic challenge, 16-week-old male $\mathrm{C} 3 \mathrm{H} / \mathrm{HeOuJ}$ mice fed a standard pellet diet (A04, Safe, Augy, France), were injected intraperitoneally with $1 \mathrm{mg} / \mathrm{kg}$ of the $\beta_{3}$-adrenergic agonist CL316,243 (C5976, Sigma-Aldrich) or vehicle $14 \mathrm{~h}$ prior to being killed.

Flow cytometry analysis Epididymal fat pad was digested by collagenase (Serlabo Technologies, Entraigues, France). Stromavascular fraction cells were incubated for $10 \mathrm{~min}$ at room temperature with FcBlock (BD Biosciences, Le Pont de Claix, France) in FACS buffer (PBS, $0.2 \%$ BSA, 2 mmol/l EDTA). Pools of specific fluorescent-labelled antibodies (PerCP-CD45, BV421-CD206, BV605-CD11c [Biolegend, San Diego, CA, USA]; FITC-F4/80 [Bio-Rad]) or Bodipy 493/503 (Invitrogen) were prepared and added to the stromavascular cell solution. Analyses were performed using a LSR Fortessa flow cytometer and DIVA software (BD Biosciences).

Immunohistochemistry analysis Epididymal fat samples were fixed in 10\% formalin (Sigma-Aldrich) for $24 \mathrm{~h}$, embedded in paraffin, sectioned and stained with F4/80 antibody
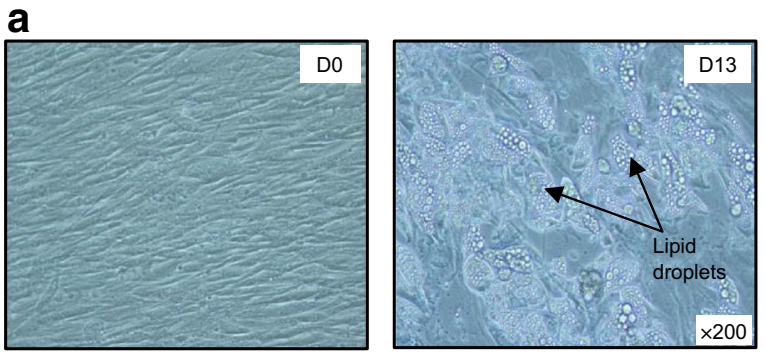

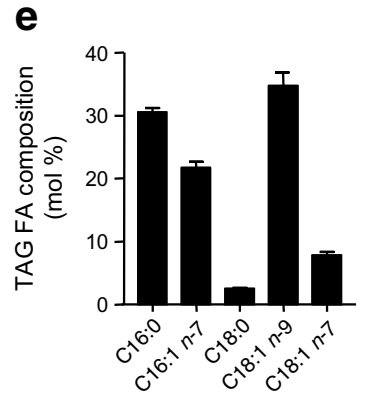

Fig. 1 Lipid metabolism in hMADS adipocytes. (a) Photomicrograph of adipocytes and (b-d) mRNA levels of de novo lipogenesis enzymes at day 0 (D0) and day 13 (D13) of differentiation. ${ }^{* *} p<0.01$ by Wilcoxon test. (e) FA composition of fat cell TAGs in differentiated adipocytes. (f) Glycerol (white bars) and NEFA (black bars) concentrations in lipolysis medium from adipocytes exposed or not exposed to $1 \times 10^{-5} \mathrm{~mol} / 1$ b

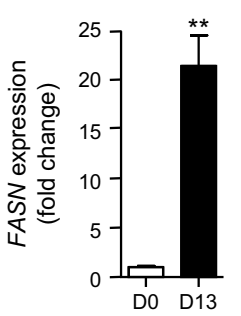

C

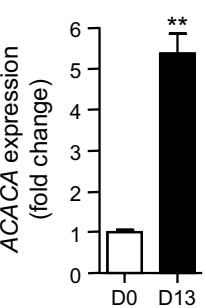

d

(AbD Serotec, Colmar, France), and revealed by an indirect peroxidase procedure. Digital images were captured using a light microscope coupled to a camera and analysed using a morphometric program (Lucia IMAGE, version 4.81; Laboratory Imaging, Prague, Czech Republic).

Measurements of mRNA levels Total RNA extraction and mRNA quantification was performed as previously described $[21,22]$. Values were normalised to the levels of $18 \mathrm{~S}$ rRNA (Applied Biosystems, Foster City, CA, USA) for murine epididymal adipose tissue, proteasome $26 \mathrm{~S}$ subunit ATPase 4 (Applied Biosystems) for THP-1 cells and LDL receptorrelated protein 10 (Applied Biosystems) for hMADS cells.

Statistics All data are presented as mean \pm SEM. GraphPad Prism Software (GraphPad Software, San Diego, CA, USA) was used for statistical analyses. Data were analysed by ANOVA with Bonferroni correction and nonparametric tests whenever appropriate.

\section{Results}

The profile of FAs released by hMADS adipocytes reflects the composition of intracellular triacylglycerol At day 13 of differentiation, hMADS cells were fully differentiated into adipocytes and had readily accumulated triacylglycerol (TAG) droplets (Fig. 1a). NEFAs and cholesteryl esters were
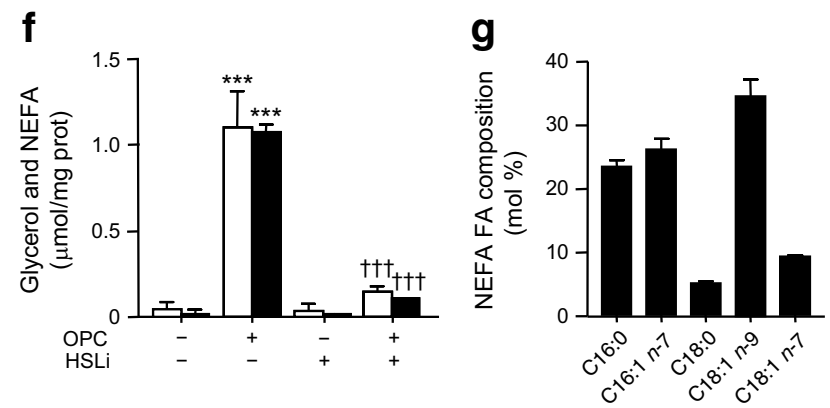

OPC3911 (OPC) and $1 \times 10^{-5} \mathrm{~mol} / 1 \mathrm{HSLi}$. $* * * p<0.001$ relative to basal condition, ${ }^{\dagger \dagger} p<0.001$ relative to the OPC condition by two-way ANOVA with Bonferroni post hoc test. Prot, protein (g) FA composition of lipolysis medium NEFAs. (b-g) Data are expressed as mean \pm SEM of three to nine different experiments for each condition 
not detected in the cellular fraction (data not shown). Differentiation was accompanied by robust induction of de novo lipogenesis gene expression (Fig. 1b-d). The major FAs found in the TAG fraction were oleic (C18:1 $n$-9), palmitic (C16:0) and palmitoleic (C16:1 $n-7)$ acids (Fig. 1e). Smaller amounts of vaccenic (C18:1 $n-7)$ and stearic (C18:0) acids were also found. The essential FAs C18:2 n-6 (linoleic acid) and C18:3 $n-3$ ( $\alpha$-linolenic acid) and $\mathrm{C} 20$ to $\mathrm{C} 22$ FAs represented $<0.5 \%$ (not shown). The FA profile of the TAG fraction reflects that expected in a case of strict de novo lipogenesis.

To investigate the effect of fat cell lipolysis on macrophage activation, we first screened for a drug with lipolytic potency on hMADS adipocytes but with minimal direct effects on THP-1 cells. Three commonly used lipolytic agents were tested: forskolin (an adenylylcyclase activator), isoprenaline (an agonist of beta-adrenergic receptors) and OPC3911 (a cyclic nucleotide phosphodiesterase 3B inhibitor). Forskolin induced the strongest lipolytic response on hMADS adipocytes (ESM Fig. 1a). However, it also potently induced IL6 and IL10 gene expression in THP-1 cells (ESM Fig. 1b,c). Isoprenaline showed a weak lipolytic action on hMADS adipocytes. It had a modest direct effect on THP-1 cell IL6 mRNA level but induced $I L 10$ gene expression as strongly as forskolin. OPC3911 readily stimulated adipocyte lipolysis with minimal direct activation of THP-1 cell inflammatory marker gene expression and was, therefore, selected as the lipolytic agent (ESM Fig. 1, Fig. 1f).

Inhibition of lipolytic induction was obtained by adding the specific hormone-sensitive lipase inhibitor (HSLi) BAY 599435. The HSLi had no effect on FA and glycerol release when used alone but decreased the lipolytic effect of OPC 3911 by $>90 \%$ (Fig. 1f). We then analysed the nature of FAs that were released in the medium in response to lipolytic stimulation (Fig. 1g). The major FAs found in the culture media were oleic, palmitic and palmitoleic acids. Vaccenic and stearic acids were also detected at lower concentrations. Thus, the profile of FAs released by hMADS adipocytes upon lipolytic stimulation largely reflected that of the TAG fraction.

\section{FAs released by hMADS adipocytes during lipolytic challenges do not induce inflammatory response in THP-} 1 macrophages The effects of hMADS cell-conditioned media on the inflammatory response in THP-1 macrophages were investigated. To validate our culture protocol and for comparison with the effects of conditioned media, THP-1 macrophages were differentiated into M1 and M2 phenotypes. Gene expression of the proinflammatory markers $I L 1 B, I L 6$ and $T N F$ were robustly increased in M1 macrophages, whereas mRNA levels of the anti-inflammatory marker $I L 10$ and the chemokine CCL2 (MCP1) were markedly induced in M2 macrophages (ESM Fig. 2a). The lipid metabolism genes FABP4 and CD36 were expressed at comparable levels in M0 and M2 macrophages that were much higher than those in M1 macrophages. Conditioned media of hMADS adipocytes with lipolytic stimulation or inhibition did not modify $I L 6 B, I L 1 B$ and $T N F$ gene expression, suggesting that adipocyte-derived lipolytic products do not induce the activation of inflammatory pathways in THP-1 cells (Fig. 2a, d, e). The lack of response to conditioned media was also observed for the M2 marker IL10 and the chemokine CCL2 (Fig. 2b, c). Indeed, when a modest change in gene expression was observed following conditioned media treatment, a similar change was observed in THP1 cells treated with hMADS cell-free control media supplemented with OPC3911. This finding suggests a direct effect of the drug on THP-1 cell gene expression. For $T N F$, and to a lesser extent for IL10 gene expression, direct induction by OPC3911 was abrogated in the presence of hMADS adipocyte-conditioned media, suggesting an effect involving a lipolysis-independent factor produced by hMADS adipocytes. Concordant data were obtained when measuring IL- 6 and MCP1 secretion in culture media (Fig. 2f, h). There was a twofold increase in IL-10 production by THP-1 cells treated with media from OPC3911-treated hMADS adipocytes (Fig. 2g). The levels were lower than those typically observed in macrophages with the anti-inflammatory M2 phenotype (ESM Fig. 2b).

The possible implication of TLR4 in the effect of FAs released from adipocyte lipolysis on macrophages was assessed by treating THP-1 macrophages with TAK-242, a specific inhibitor of the TLR4 signalling pathway [23]. First, we checked that TLR4 was functional and that TAK-242 was efficient in THP-1 cells treated with lipopolysaccharide (LPS), the prototypical TLR4 ligand (ESM Fig. 3a). However, TAK-242 had no significant effect on inflammation-related marker gene expression in THP-1 cells treated with conditioned media from OPC3911-stimulated hMADS adipocytes (ESM Fig. 3b). These data show that lipolytic products, FAs or other molecules, do not activate TLR4 signalling pathways stimulating inflammation-related gene expression.

We next investigated the direct effect of mixtures of FAs mimicking the FA concentration and composition either of media from hMADS fat cells stimulated with a lipolytic agent, i.e. containing FAs synthesised by de novo lipogenesis, or of ex vivo lipolysis from human AT pieces, i.e. including FAs from dietary sources (ESM Table 1) [19]. Compared with the FA-free control, treatment of THP-1 macrophages with the FA mixtures induced no change in IL6,IL10,IL1B and TNF gene expression (Fig. 3a, b, d, e). A modest effect was seen on CCL2 mRNA levels (Fig. 3c) that was much lower than the induction observed during differentiation into M2 macrophages (ESM Fig. 2a). Treatment with palmitate but not with oleate increased mRNA levels of IL6,IL10,CCL2, ILIB and TNF (Fig. 3a-e), as well as IL-6, IL-10 and MCP1 production in culture media (Fig. $3 \mathrm{f}-\mathrm{h}$ ). 
a

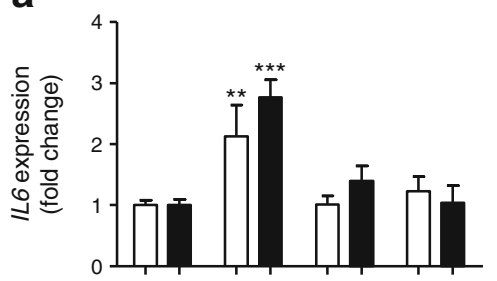

b

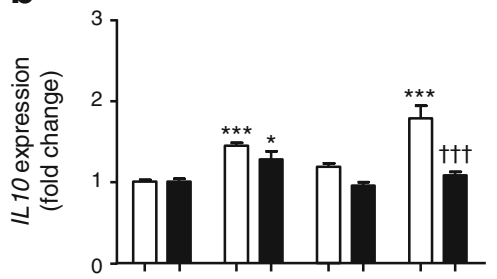

C

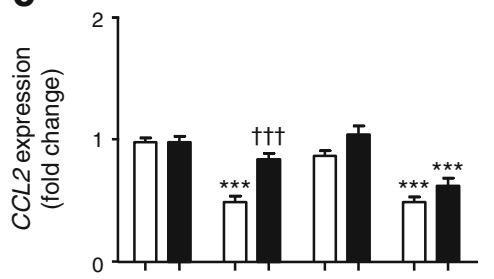

d

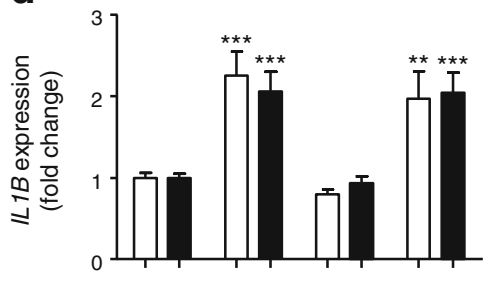

e

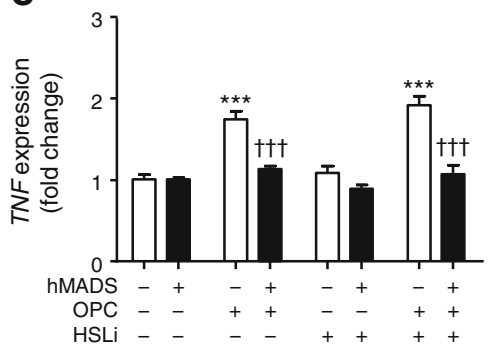

f

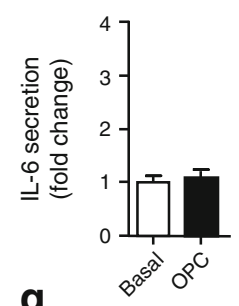

g
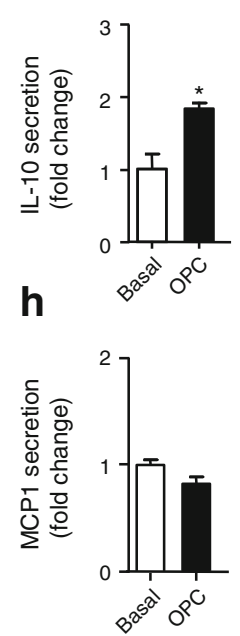

a

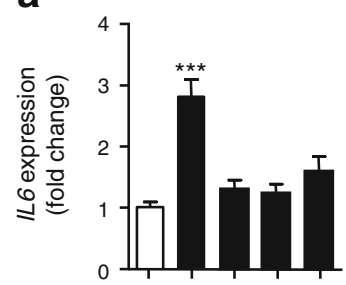

b

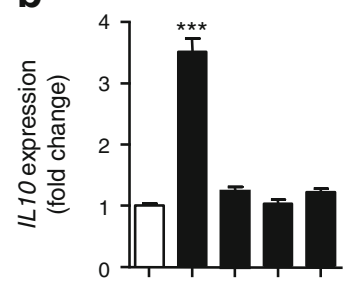

C

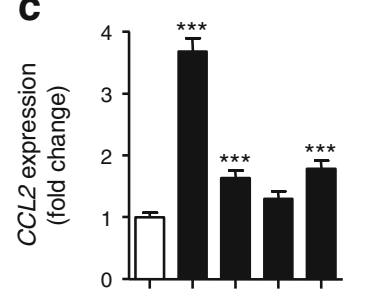

d

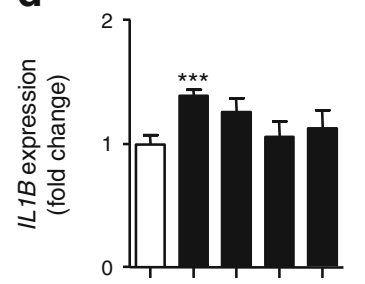

e

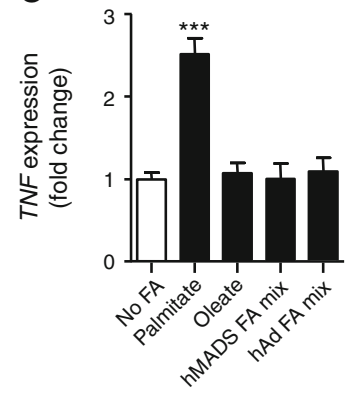

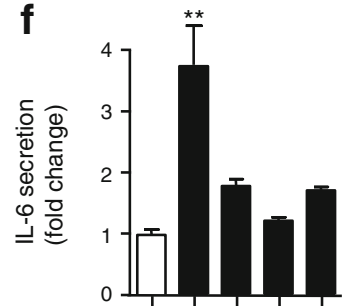

g

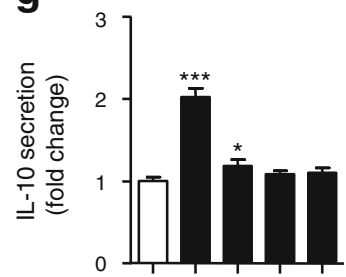

h

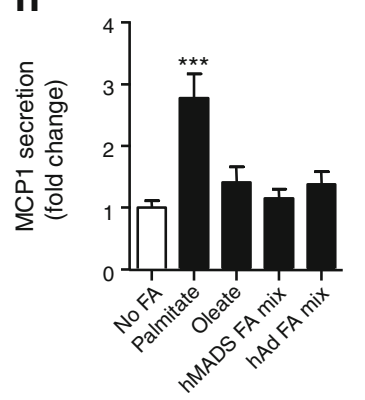

Fig. 2 Effects of adipocyte lipolysis conditioned media on (a-e) gene expression and (f-h) production of ILs and chemokine by THP-1 macrophages. Macrophages were treated with conditioned media from nonstimulated, OPC3911 (OPC) and/or HSLi-stimulated-hMADS (black bars) or exposed directly with drugs (white bars). ${ }^{*} p<0.05$, ${ }^{*} p<0.01$, $* * * p<0.001$ relative to the control condition without treatment; ${ }^{\dagger} p<0.001$ relative to the condition without hMADS conditioned media by two-way ANOVA with Bonferroni post hoc test or Wilcoxon test. Data are expressed as mean \pm SEM of six different experiments for each condition

THP-1 macrophages readily store TAG in the presence of adipocyte lipolysis-derived FA Upon treatment with lipolysis-induced conditioned media, THP-1 macrophages accumulated intra-cytoplasmic droplets that gave them the

Fig. 3 Effects of exogenous FA mixtures on gene expression (a-e) and (f-h) production of ILs and chemokine by THP-1 macrophages. Macrophages were treated with vehicle (no FA; white bars), $350 \mathrm{mmol} / \mathrm{l}$ of palmitate, oleate, hMADS adipocyte lipolysis (hMADS FA mix) or human adipocyte lipolysis (hAd FA mix) FA mixtures (black bars). ${ }^{*} p<0.05,{ }^{*} p<0.01, * * * p<0.001$ relative to the control condition without treatment (white bars) by one-way ANOVA with Bonferroni post hoc test. Data are expressed as mean $\pm \mathrm{SEM}$ of nine different experiments for each condition

appearance of foam cells. These cells were stained with Bodipy, revealing that the droplets were filled with neutral lipids (Fig. 4a). Untreated THP-1 macrophages and 
a
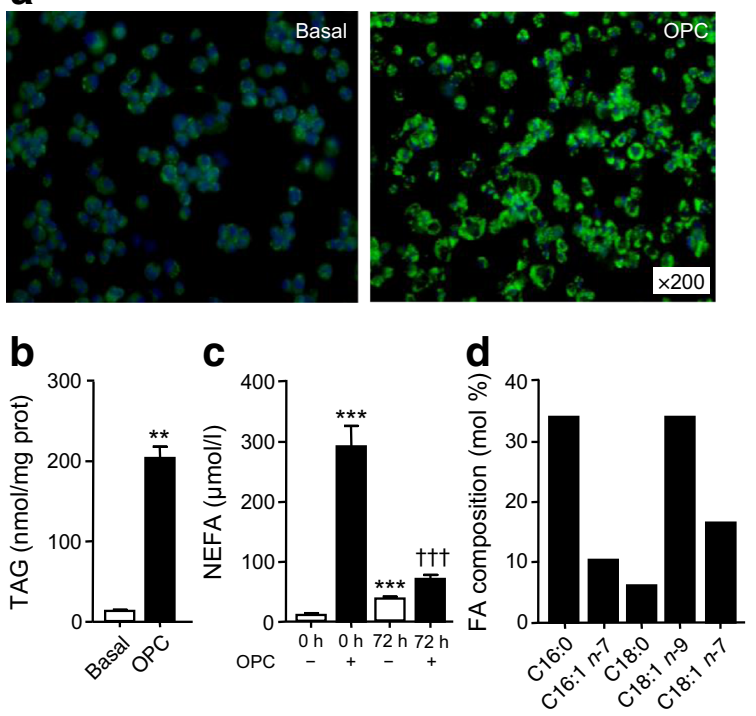

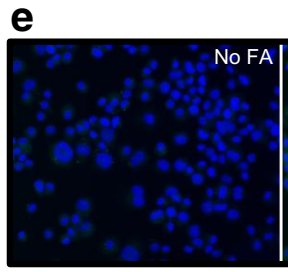

f

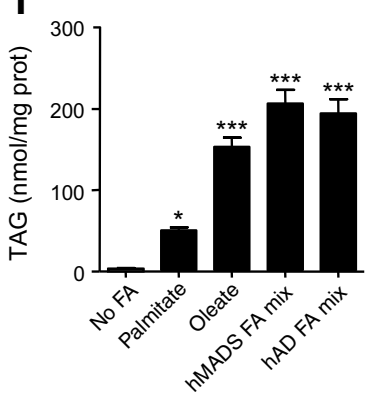

g

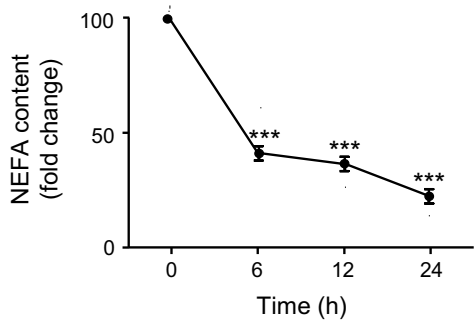

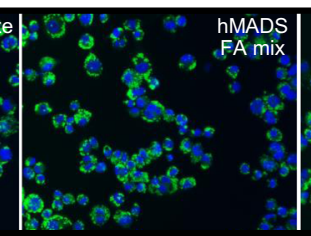

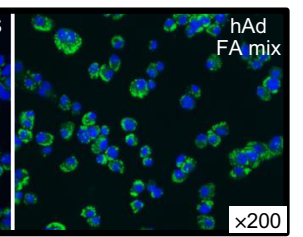

$\mathbf{h}$

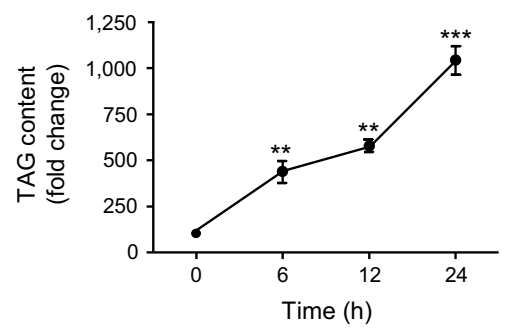

Fig. 4 Effects of hMADS adipocyte lipolysis conditioned media and exogenous FA mixtures on lipid storage in THP-1 macrophages. (a) Fluorescent microscopy of neutral lipid accumulation in macrophages exposed to conditioned media from non-stimulated (basal) or OPC3911-stimulated (OPC) hMADS. Neutral lipid droplets show green fluorescence. (b) TAG content in macrophages exposed to conditioned media from non-stimulated (basal) or OPC-stimulated adipocytes. $* * p<0.01$ relative to control condition by Wilcoxon test. Prot, protein (c) NEFA concentration in the macrophage culture medium before and after $72 \mathrm{~h}$ of treatment with conditioned media from non-stimulated (-) or OPC-stimulated $(+)$ adipocytes. ${ }^{* * *} p<0.001$ relative to NEFA concentration at time 0 before THP-1 macrophage exposure with conditioned media from non-stimulated hMADS. ${ }^{\dagger \dagger} p<0.001$ relative to NEFA concentration at time 0 before macrophage exposure with conditioned media from OPC3911-stimulated hMADS by one-way ANOVA with

macrophages treated with unstimulated conditioned media contained few intracellular stained structures, whereas abundant staining was observed in THP-1 macrophages treated with conditioned media from OPC3911-treated adipocytes. To determine the nature of the neutral lipids stored in the
Bonferroni post hoc test. (d) FA composition of the macrophage TAG fraction after $72 \mathrm{~h}$ of treatment with conditioned media from OPC-stimulated adipocytes. (e) Fluorescent microscopy of neutral lipid accumulation in macrophages treated with vehicle (no FA) and $350 \mathrm{mmol} / \mathrm{l}$ of palmitate (Palm), oleate, in vitro hMADS adipocyte lipolysis (hMADS FA mix) or ex vivo human adipocyte lipolysis (hAd FA mix) FA mixtures. Neutral lipid droplets and nuclei show green and blue fluorescence, respectively. (f) TAG content in macrophages exposed to FA mixtures. ${ }^{*} p<0.05, * * * p<0.001$ relative to no FA condition by one-way ANOVA with Bonferroni post hoc test. Prot, protein. Time course of (g) NEFA concentrations in culture media and (h) TAG content of macrophages exposed to hMADS adipocyte lipolysis FA mixture. ${ }^{* *} p<0.01$, $*_{* *} p 0.001$ relative to T0 by one-way ANOVA with Bonferroni post hoc test. Data are expressed as mean \pm SEM of three to nine different experiments for each condition

droplets, we analysed the lipid composition of the cellular fractions of THP-1 cells. Neutral lipids accumulating in THP-1 macrophages were TAG (Fig. 4b). At the same time, NEFA concentration in the culture medium from THP-1 cells exposed to conditioned media from OPC3911-treated 
adipocytes drastically fell after $72 \mathrm{~h}$ of culture (Fig. 4c). Conditioned media from OPC3911-treated adipocytes increased gene expression of lipid transport and storage molecules, ADRP (also known as PLIN2) and CD36, in THP1 cells (ESM Fig. 4). Overall, the FA profile of the THP-1 TAG subfraction (Fig. 4d) reflected that of both the lipolysis conditioned media (Fig. 1e) and the hMADS adipocyte TAG (Fig. 1c). When cultured in medium supplemented with FA mixtures, THP-1 macrophages exhibited plentiful intracellular Bodipy-stained structures that were not observed in THP-1 cells maintained in FA-free conditions (Fig. 4e). TAG accumulation was similar using oleate alone, FA mixtures containing de novo lipogenesisor diet-derived FAs, and much higher than when palmitate alone was added to macrophages (Fig. 4e, f). The two FA mixtures stimulated gene expression of $A D R P$ and $C D 36$ (ESM Fig. 4). Using de novo lipogenesis-derived FA mixture, TAG content gradually increased with time in THP-1 cells, whereas NEFA level in the media decreased in a concomitant manner (Fig. 4g, h).
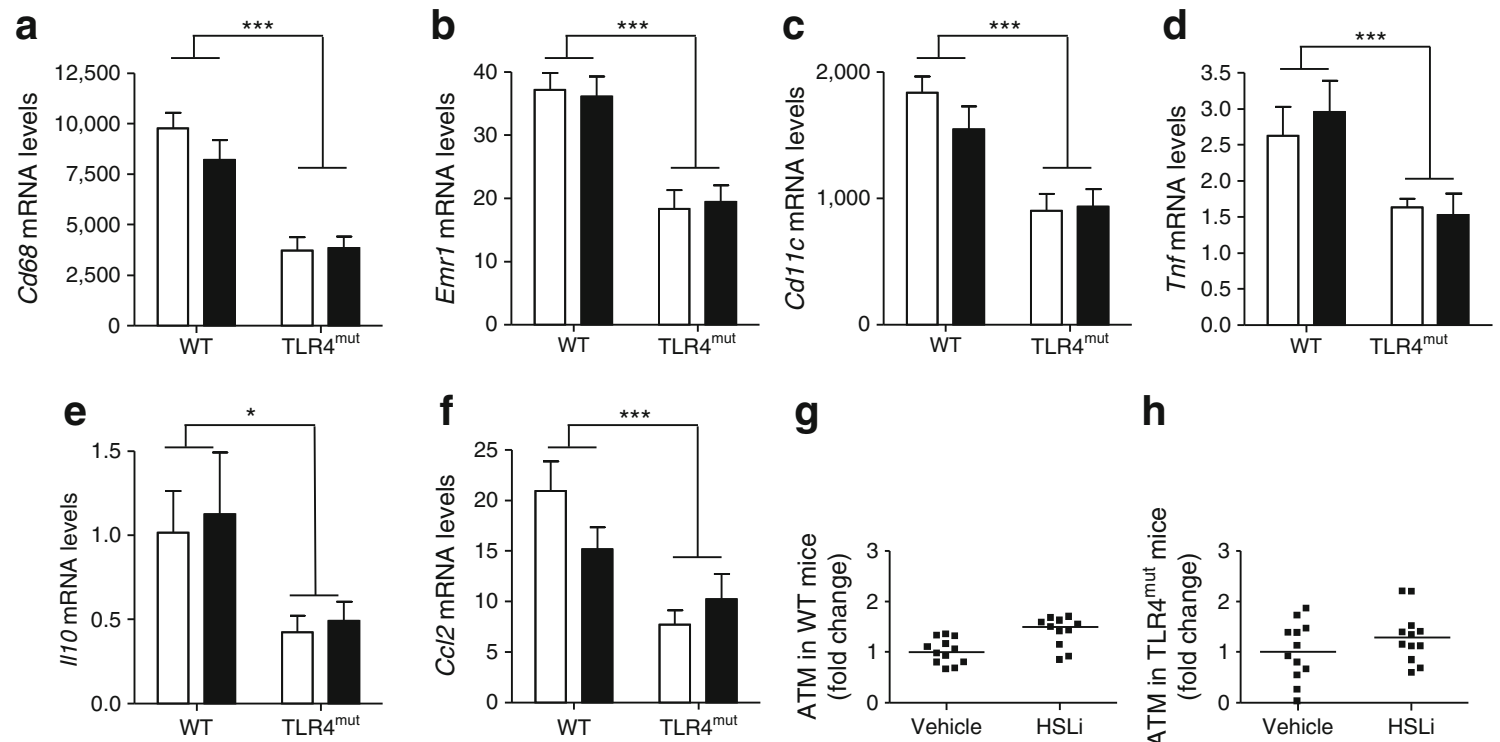

g
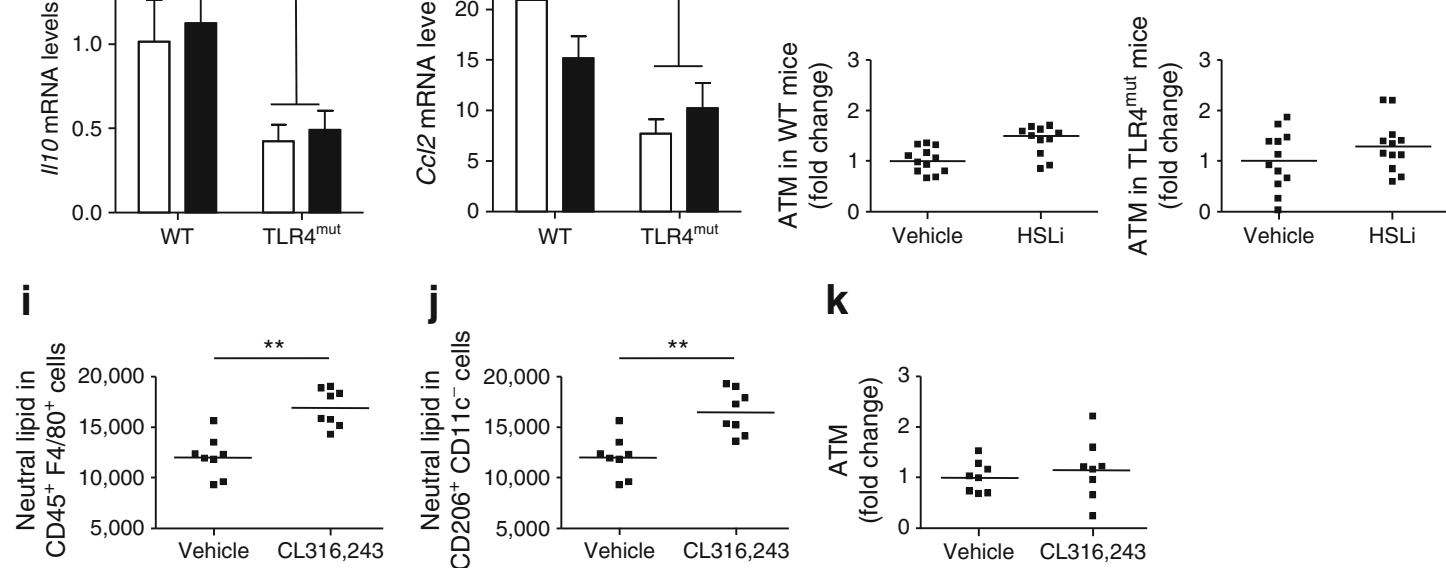

$\mathbf{K}$

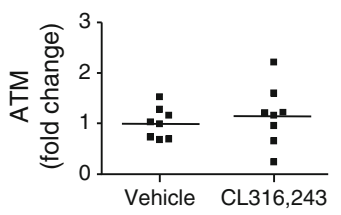

Fig. 5 In vivo effect of chronic inhibition and acute stimulation of lipolysis on ATMs. (a-f) mRNA levels in epididymal AT from $\mathrm{C} 3 \mathrm{H} / \mathrm{HeJ}$ carrying a mutation of TLR4 (TLR $4^{\mathrm{mut}}$ ) and $\mathrm{C} 3 \mathrm{H} / \mathrm{HeOuJ}$ (WT) obese male mice treated with vehicle (white bars) or HSLi for 2 weeks (black bars); 12 animals per group. ${ }^{*} p<0.05, * * * p<0.001$ between WT and TLR4 ${ }^{\text {mut }}$ mice by two-way ANOVA with Bonferroni post hoc test. (g, h) Quantitation of ATMs (CD45 $5^{+} 4 / 80^{+}$cells) by flow cytometry. (i, j)

In vivo modulation of lipolysis impacts lipid accumulation but not inflammation in ATMs In mice, lipid mobilisation from fat stores is maximal during the daily resting phase. To investigate the in vivo relationship between AT lipolysis and inflammation, we treated two strains of high-fat diet-fed mice in the morning with the antilipolytic drug HSLi for 15 days. $\mathrm{C} 3 \mathrm{H} / \mathrm{HeOuJ}$ mice have intact TLR4 signalling (WT), whereas $\mathrm{C} 3 \mathrm{H} / \mathrm{HeJ}$ mice show defective TLR4 signalling (TLR $4^{\mathrm{mut}}$ ) [24]. The chronic inhibition of lipolysis was shown by a robust decrease in plasma glycerol levels in the two strains (ESM Fig. 5a). Gene expression of ATM markers such as CD68 (Cd68), F4/80 (Emr1 also known as Adgrel) and CD11c (Cd11c also known as Itgax) was significantly lower in TLR $4{ }^{\text {mut }}$ mice than in WT mice (Fig. 5a-c). Deficiency in TLR4 signalling also showed lower mRNA levels of pro(Tnf) and anti- (Il10) inflammatory markers and of the chemokine MCP1 (Ccl2) (Fig. 5d-f). Chronic inhibition of lipolysis had no effect on ATM- and inflammation-related marker gene expression. Accordingly, treatment with HSLi did not modify ATM content determined by flow cytometry (Fig. $5 \mathrm{~g}, \mathrm{~h}$ ).
Neutral lipid (Bodipy mean fluorescence) in AT total macrophages $\left(\mathrm{CD} 45^{+} \mathrm{F} 4 / 80^{+}\right.$cells) and $\mathrm{M} 2$ macrophages $\left(\mathrm{CD} 45^{+} \mathrm{F} 4 /\right.$ $80^{+} \mathrm{CD} 206^{+} \mathrm{CD} 11 \mathrm{c}^{-}$) of male $\mathrm{C} 3 \mathrm{H} / \mathrm{HeOuJ}$ mice treated with vehicle or lipolytic agent CL 316,243 for $14 \mathrm{~h} .{ }^{* *} p<0.01$ between the two groups by Mann-Whitney test. (k) Quantitation of ATMs (CD45 $5^{+} \mathrm{F} 4 / 80^{+}$cells) by flow cytometry. (g-k) Squares indicate data from individual mice and lines indicate means 
To show in vivo lipid accumulation in ATMs consecutive with stimulated fat cell lipolysis, $\mathrm{C} 3 \mathrm{H} / \mathrm{HeOuJ}$ mice were injected with the $\beta_{3}$-adrenergic agonist CL316.243. An increase in plasma glycerol level was observed at 6 and $14 \mathrm{~h}$ post-injection (ESM Fig 5b). At $14 \mathrm{~h}$ after injection of the lipolytic drug, there was a robust accumulation of neutral lipids in ATMs (Fig. 5i, j). This increase occurred without changes in ATM number (Fig. 5k). There was no modification of ATM phenotypes, i.e. the percentage of $\mathrm{CD} 45^{+} \mathrm{F} 4 /$ $80^{+} \mathrm{CD} 206^{-} \mathrm{CD} 11 \mathrm{c}^{+}, \mathrm{CD} 45^{+} \mathrm{F} 4 / 80^{+} \mathrm{CD} 206^{+} \mathrm{CD} 11 \mathrm{c}^{-}$, $\mathrm{CD} 45^{+} \mathrm{F} 4 / 80^{+} \mathrm{CD} 206^{+} \mathrm{CD} 11 \mathrm{c}^{+}$and $\mathrm{CD} 45^{+} \mathrm{F} 4 /$ $80^{+} \mathrm{CD} 206^{-} \mathrm{CD} 11 \mathrm{c}^{-}$cells was unchanged (data not shown). Immunohistochemistry of AT sections showed no difference between vehicle and treated animals in the low number of crown-like structures, i.e. macrophages scavenging lipids released by necrotic adipocytes (ESM Fig. 5c).

\section{Discussion}

In the present study, we investigated the effects of adipocyte lipolysis products, notably FAs, on human ATM activation. During lipolysis, FAs and other molecules are released from fat cells. Using in vitro and in vivo approaches, we showed that stimulation or inhibition of adipocyte lipolysis does not modulate the inflammationrelated response of macrophages. Our results also rule out a role for TLR4, a receptor of innate immunity activated by saturated FA, in lipolysis-derived FA activation of macrophages. ATMs, however, play a role in scavenging FAs released by fat cell lipolysis. Our data suggest that, in usual dietary conditions, FAs released from fat cells are unlikely to be major determinants of ATM activation.

Mouse studies suggest that saturated FAs induce a proinflammatory response by ATMs [12-14, 25]. These effects are mostly dependent on TLR4. However, some studies argue against a direct activation of TLR4 signalling by saturated FAs [26, 27]. Recently, evidence for resolution of this paradox was provided. Fetuin-A, a liver-derived glycoprotein, acts as an adaptor between saturated FAs and TLR4 allowing activation of intracellular signal transduction pathways [28, 29]. In our in vitro experiments, the concentration of fetuin-A provided by FBS was sufficient to ensure maximal effect of the adaptor protein [29]. Indeed, addition of the saturated FA, palmitate, elicited an inflammatory response on macrophages. Therefore, the lack of immune response induced by FA mixtures similar in composition either to lipolytic media containing FA derived from de novo lipogenesis [30] or to ex vivo human AT lipolysis containing FA derived from the diet [19, 31] cannot be ascribed to the absence of an adaptor protein. Instead, the concentration and nature of the FAs released during lipolysis may explain the lack of effect. The concentration of palmitate required to elicit activation of macrophages is higher than that found during AT lipolysis [29, 32]. Monounsaturated FAs do not stimulate TLR4-mediated activation of nuclear factor- $\mathrm{KB}(\mathrm{NF}-\mathrm{kB})[29,33]$. Unsaturated FAs have been shown to inhibit the LPS-induced NF- $\mathrm{KB}$ signalling pathway in macrophages [25, 34]. Moreover, monounsaturated FAs, such as oleate, protect against various lipotoxic effects of palmitate [35-39]. The protective effects of oleate are reported at concentrations lower or equimolar to that of palmitate. In lipolysis-derived FA mixtures, the concentration of oleate is higher than that of palmitate and the ratio of monounsaturated to saturated FAs is above 1 . Therefore, it is likely that the concentration of saturated FAs and the ratio of monounsaturated to saturated FAs produced during adipocyte lipolysis concur with the absence of an inflammatory response in macrophages. In vivo, acute stimulation and chronic inhibition of AT lipolysis did not modulate the AT immune response. Consistent with in vitro data, the mouse studies show that TLR4 was not involved in this adaptation. In vivo, stimulation of lipolysis promoted accumulation of neutral lipids in ATMs without change in number and phenotypes. Lipid droplet accumulation into ATMs occurred without a change in the number of crown-like structures that result from the recruitment of macrophages around dead adipocytes, which is a TLR4-dependent mechanism [24, 40, 41]. These data point to the presence of lipids from dead adipocytes rather than to FAs released from lipolysis in eliciting the proinflammatory response through TLR4-dependent signalling.

AT lipolysis-derived FAs were readily stored into macrophage TAGs as multilocular lipid droplets. The rapid fall in NEFA concentrations in the medium demonstrates the propensity of macrophages to efficiently absorb local excess of lipids. Accordingly, mouse ATMs accumulate lipids in response to lipolysis during fasting [42]. Here, we showed that this accumulation occurs during acute stimulation of lipolysis. The data indicate a role for ATMs as a buffer for local increase in FA concentrations. The uptake and metabolism of FAs in macrophages may modify their phenotype as shown in several recent studies [43, 44]. Along with this hypothesis, in vivo impairment of FA catabolism in ATMs that have accumulated lipids has systemic and local consequences [45]. However, long term lipid accumulation in ATMs promotes M1 polarisation and AT inflammation [46]. Together, these results suggest there is no association between fat cell lipolysisderived FA uptake and subsequent storage as TAGs in ATMs and the induction of inflammatory pathways.

In summary, FAs released during AT lipolysis do not induce TLR4-mediated production of cytokines, chemokines and ILs by ATMs. Instead, macrophages scavenge FAs in excess and convert them into TAG stored within multilocular lipid droplets. This may reflect an adaptive function of ATMs to preserve AT homeostasis in times of moderate- or short- 
term increase in lipid flux within AT, a mechanism that might be overridden with excess supply of saturated fat in the diet.

Acknowledgements We gratefully acknowledge the GenoToul Animal Care, Anexplo, Quantitative Transcriptomic (F. Martins and J. J. Maoret) facility. We especially thank M. Combes (Obesity Research Laboratory, I2MC) for expert technical assistance. DL is a member of Institut Universitaire de France.

Funding This work was supported by grants from Fondation pour la Recherche Médicale (to DL), Agence Nationale de la Recherche (LIPOB and OBELIP projects to DL), Région Midi-Pyrénées (to DL) and the Commission of the European Communities (Projects DioGenes, ADAPT and DIABAT to DL).

Duality of interest The authors declare that there is no duality of interest associated with this manuscript.

Contribution statement $\mathrm{SCB}, \mathrm{CIK}$, DL designed the research, analysed the data and wrote the manuscript. SCB, CIK, CL, IV, EM, DB, GT, MAM, AZG, CP, MH, LM, SN, CM performed the present study, contributed to analysis and interpretation of data and to critical revision of the manuscript. All authors approved the version to be published. DL is the guarantor of this work.

\section{References}

1. Lumeng CN, Saltiel AR (2011) Inflammatory links between obesity and metabolic disease. J Clin Invest 121:2111-2117

2. Olefsky JM, Glass CK (2010) Macrophages, inflammation, and insulin resistance. Annu Rev Physiol 72:219-246

3. Weisberg SP, McCann D, Desai M, Rosenbaum M, Leibel RL, Ferrante AW Jr (2003) Obesity is associated with macrophage accumulation in adipose tissue. J Clin Invest 112:1796-1808

4. Xu H, Barnes GT, Yang Q et al (2003) Chronic inflammation in fat plays a crucial role in the development of obesity-related insulin resistance. J Clin Invest 112:1821-1830

5. Hotamisligil GS, Peraldi P, Budavari A, Ellis R, White MF, Spiegelman BM (1996) IRS-1-mediated inhibition of insulin receptor tyrosine kinase activity in TNF-alpha- and obesity-induced insulin resistance. Science 271:665-668

6. Kanda H, Tateya S, Tamori Y et al (2006) MCP-1 contributes to macrophage infiltration into adipose tissue, insulin resistance, and hepatic steatosis in obesity. J Clin Invest 116:1494-1505

7. Duncan RE, Ahmadian M, Jaworski K, Sarkadi-Nagy E, Sul HS (2007) Regulation of lipolysis in adipocytes. Annu Rev Nutr 27: 79-101

8. Langin D, Arner P (2006) Importance of TNFalpha and neutral lipases in human adipose tissue lipolysis. Trends Endocrinol Metab 17:314-320

9. Samuel VT, Petersen KF, Shulman GI (2010) Lipid-induced insulin resistance: unravelling the mechanism. Lancet 375:2267-2277

10. Suganami T, Nishida J, Ogawa Y (2005) A paracrine loop between adipocytes and macrophages aggravates inflammatory changes: role of free fatty acids and tumor necrosis factor alpha. Arterioscler Thromb Vasc Biol 25:2062-2068

11. Lee JY, Sohn KH, Rhee SH, Hwang D (2001) Saturated fatty acids, but not unsaturated fatty acids, induce the expression of cyclooxygenase-2 mediated through Toll-like receptor 4. J Biol Chem 276:16683-16689

12. Nguyen MT, Favelyukis S, Nguyen AK et al (2007) A subpopulation of macrophages infiltrates hypertrophic adipose tissue and is activated by free fatty acids via Toll-like receptors 2 and 4 and JNKdependent pathways. J Biol Chem 282:35279-35292

13. Shi H, Kokoeva MV, Inouye K, Tzameli I, Yin H, Flier JS (2006) TLR4 links innate immunity and fatty acid-induced insulin resistance. J Clin Invest 116:3015-3025

14. Suganami T, Tanimoto-Koyama K, Nishida J et al (2007) Role of the Toll-like receptor 4/NF-kappaB pathway in saturated fatty acidinduced inflammatory changes in the interaction between adipocytes and macrophages. Arterioscler Thromb Vasc Biol 27:84-91

15. Tsukumo DM, Carvalho-Filho MA, Carvalheira JB et al (2007) Loss-of-function mutation in Toll-like receptor 4 prevents dietinduced obesity and insulin resistance. Diabetes 56:1986-1998

16. Bezaire V, Mairal A, Ribet $C$ et al (2009) Contribution of adipose triglyceride lipase and hormone-sensitive lipase to lipolysis in hMADS adipocytes. J Biol Chem 284:18282-18291

17. Tavernier G, Galitzky J, Valet P et al (1995) Molecular mechanisms underlying regional variations of catecholamine-induced lipolysis in rat adipocytes. Am J Physiol 268:E1135-E1142

18. Langin D, Dicker A, Tavernier G et al (2005) Adipocyte lipases and defect of lipolysis in human obesity. Diabetes 54:3190-3197

19. Raclot T, Langin D, Lafontan M, Groscolas R (1997) Selective release of human adipocyte fatty acids according to molecular structure. Biochem J 324:911-915

20. Caspar-Bauguil S, Fioroni A, Galinier A et al (2012) Proinflammatory phospholipid arachidonic acid/eicosapentaenoic acid ratio of dysmetabolic severely obese women. Obes Surg 22:935944

21. Girousse A, Tavernier G, Valle C et al (2013) Partial inhibition of adipose tissue lipolysis improves glucose metabolism and insulin sensitivity without alteration of fat mass. PLoS Biol 11:e1001485

22. Viguerie N, Montastier E, Maoret JJ et al (2012) Determinants of human adipose tissue gene expression: impact of diet, sex, metabolic status, and cis genetic regulation. PLoS Genet 8:e1002959

23. Ii M, Matsunaga N, Hazeki K et al (2006) A novel cyclohexene derivative, ethyl (6R)-6-[N-(2-Chloro-4-fluorophenyl)sulfamoyl]cyclohex1-ene-1-carboxylate (TAK-242), selectively inhibits toll-like receptor 4-mediated cytokine production through suppression of intracellular signaling. Mol Pharmacol 69:1288-1295

24. Vila IK, Badin PM, Marques MA et al (2014) Immune cell Toll-like receptor 4 mediates the development of obesity- and endotoxemiaassociated adipose tissue fibrosis. Cell Rep 7:1116-1129

25. Lee JY, Plakidas A, Lee WH et al (2003) Differential modulation of Toll-like receptors by fatty acids: preferential inhibition by n-3 polyunsaturated fatty acids. J Lipid Res 44:479-486

26. Erridge C, Samani NJ (2009) Saturated fatty acids do not directly stimulate Toll-like receptor signaling. Arterioscler Thromb Vasc Biol 29:1944-1949

27. Schaeffler A, Gross P, Buettner R et al (2009) Fatty acid-induced induction of Toll-like receptor-4/nuclear factor-kappaB pathway in adipocytes links nutritional signalling with innate immunity. Immunology 126:233-245

28. Heinrichsdorff J, Olefsky JM (2012) Fetuin-A: the missing link in lipid-induced inflammation. Nat Med 18:1182-1183

29. Pal D, Dasgupta S, Kundu R et al (2012) Fetuin-A acts as an endogenous ligand of TLR4 to promote lipid-induced insulin resistance. Nat Med 18:1279-1285

30. Collins JM, Neville MJ, Pinnick KE et al (2011) De novo lipogenesis in the differentiating human adipocyte can provide all fatty acids necessary for maturation. J Lipid Res 52:1683-1692

31. Hodson L, Skeaff CM, Fielding BA (2008) Fatty acid composition of adipose tissue and blood in humans and its use as a biomarker of dietary intake. Prog Lipid Res 47:348-380

32. Huang S, Rutkowsky JM, Snodgrass RG et al (2012) Saturated fatty acids activate TLR-mediated proinflammatory signaling pathways. J Lipid Res 53:2002-2013 
33. Dasu MR, Jialal I (2011) Free fatty acids in the presence of high glucose amplify monocyte inflammation via Toll-like receptors. Am J Physiol Endocrinol Metab 300:E145-E154

34. Chang CF, Chau YP, Kung HN, Lu KS (2012) The lipopolysaccharide-induced pro-inflammatory response in RAW264.7 cells is attenuated by an unsaturated fatty acid-bovine serum albumin complex and enhanced by a saturated fatty acidbovine serum albumin complex. Inflamm Res 61:151-160

35. Collins JM, Neville MJ, Hoppa MB, Frayn KN (2010) De novo lipogenesis and stearoyl-CoA desaturase are coordinately regulated in the human adipocyte and protect against palmitate-induced cell injury. J Biol Chem 285:6044-6052

36. Ishiyama J, Taguchi R, Akasaka Y et al (2011) Unsaturated FAs prevent palmitate-induced LOX-1 induction via inhibition of ER stress in macrophages. J Lipid Res 52:299-307

37. Kadotani A, Tsuchiya Y, Hatakeyama H, Katagiri H, Kanzaki M (2009) Different impacts of saturated and unsaturated free fatty acids on COX-2 expression in $\mathrm{C}(2) \mathrm{C}(12)$ myotubes. Am J Physiol Endocrinol Metab 297:E1291-E1303

38. L'Homme L, Esser N, Riva L et al (2013) Unsaturated fatty acids prevent activation of NLRP3 inflammasome in human monocytes/ macrophages. J Lipid Res 54:2998-3008

39. Staiger K, Staiger H, Weigert C, Haas C, Haring HU, Kellerer M (2006) Saturated, but not unsaturated, fatty acids induce apoptosis of human coronary artery endothelial cells via nuclear factorkappaB activation. Diabetes 55:3121-3126
40. Cinti S, Mitchell G, Barbatelli G et al (2005) Adipocyte death defines macrophage localization and function in adipose tissue of obese mice and humans. J Lipid Res 46:2347-2355

41. Saberi M, Woods NB, de Luca C et al (2009) Hematopoietic cellspecific deletion of Toll-like receptor 4 ameliorates hepatic and adipose tissue insulin resistance in high-fat-fed mice. Cell Metab 10:419-429

42. Kosteli A, Sugaru E, Haemmerle G et al (2010) Weight loss and lipolysis promote a dynamic immune response in murine adipose tissue. J Clin Invest 120:3466-3479

43. Huang SC, Everts B, Ivanova Y et al (2014) Cell-intrinsic lysosomal lipolysis is essential for alternative activation of macrophages. Nat Immunol 15:846-855

44. Klein-Wieringa IR, Andersen SN, Kwekkeboom JC et al (2013) Adipocytes modulate the phenotyped of human macrophages through secreted lipids. J Immunol 191:1356-1363

45. Xu X, Grijalva A, Skowronski A, van Eijk M, Serlie MJ, Ferrante AW Jr (2013) Obesity activates a program of lysosomal-dependent lipid metabolism in adipose tissue macrophages independently of classic activation. Cell Metab 18:816-830

46. Prieur X, Mok CY, Velagapudi VR et al (2011) Differential lipid partitioning between adipocytes and tissue macrophages modulates macrophage lipotoxicity and M2/M1 polarization in obese mice. Diabetes 60:797-809 